\title{
Influence of Porosity on the Deformation Behaviour of Systems with Nanostructured Thermal Barier Coatings
}

\author{
D. D. Moiseenko ${ }^{1, \text { a) }}$, P. V. Maksimov ${ }^{1}$, V. E. Panin ${ }^{1,2}$, V. P. Sergeev ${ }^{1,2}$, \\ S. V. Panin ${ }^{1,2}$, and F. Berto ${ }^{3}$ \\ ${ }^{1}$ Institute of Strength Physics and Materials Science SB RAS, Tomsk, 634055, Russia \\ ${ }^{2}$ National Research Tomsk Polytechnic University, Tomsk, 634050, Russia \\ ${ }^{3}$ University of Padova, Vicenca, 36100, Italy \\ a) Corresponding author: mdd@ispms.tsc.ru
}

\begin{abstract}
Based on the principal concepts of physical mesomechanics that take into account reversible structural-phase transformations in the rotational-wave flows at the interfaces, a new modification of the multilevel discrete-continuous method of excitable cellular automata (ECA) has been developed. The new modification explicitly takes into account the porosity and the nanocrystalline structure. Also, algorithms for calculating the local moments of forces and the angular velocities of microrotations arising in a structurally heterogeneous medium have been implemented. The model has been complemented by the dissipation conditions expressed for mechanical energy flows. On the basis of the method of excitable cellular automata, some numerical experiments on thermal loading of three-layered compositions with the intermediate layers of different structures have been carried out. It is shown that nanostructuring of the intermediate sublayer and the introduction of nanoporosity gives rise to a positive effect on the relaxation ability of thermal barrier coatings.
\end{abstract}

\section{INTRODUCTION}

One of the key factors to affect the service life of the engines for spacecraft industry is the stability of the structure under high temperatures and the resistance of thermal barrier coatings. Nowadays this topic is of particular importance both in experimental and theoretical studies. Some results of the authors on thermal barrier coatings design might be found in [1]. The latter describes the investigation data aimed at increasing the operating temperature on the surface of the combustion chamber parts as well as the appropriate value of the temperature gradient across its cross-section under actual operating conditions. The porosity of multilayered coatings (including nanoscale ones) is of great importance in terms of crack growth resistance. The complexity and diversity of the processes induced by local thermal impact is accompanied by both structural transformations and deformation. This is the underlying motivation for the development of modern methods of multilevel simulation of such processes.

In physical mesomechanics of materials [2], a theory of the evolution of stress moment fields that initiates reversible structural phase transformations and the formation of vortex structures at the interfaces is developed. In doing so, a material under consideration is presented as a set of a 3D crystalline system and a 2D subsystem of all interfaces along which the rotation wave flows of mass and energy are generated.

In particular, rotation-wave flows actively manifest themselves under dynamic loading, and especially under thermal impact. Rotational modes of deformation arise as a result of influence of local moments of force. For the sake of computer simulation of the generation and subsequent evolution of the local force moment fields to occur under intensive dynamic loading, a discrete-continuum method of excitable cellular automata (ECA) was developed. Cellular automata can be divided into three types: bistable, excitable, oscillatory. An element of bistable automata can take one of the two possible states. An excitable element is capable of following a set (a chain) of switchable states under external influence. Oscillatory elements can also possess a set of possible states, and enter them without an impact from the outside. Based on the features of the processes being simulated to accept the redistribution of

International Conference on Physical Mesomechanics of Multilevel Systems 2014

AIP Conf. Proc. 1623, 423-426 (2014); doi: 10.1063/1.4898972

(C) 2014 AIP Publishing LLC 978-0-7354-1260-6/ $\$ 30.00$ 
thermal and mechanical energy in a material, excitable cellular automaton was selected as the modeling tool. Each element of the automaton is characterized by a certain set of neighbors being located on the first coordination sphere, as well as the numerical parameters of the relevant material to be contained in the simulated volume. Those include the elastic modulus, density, shear modulus, dislocation density, thermal conductivity, specific heat, thermal expansion coefficient, etc. Upon interaction with adjacent elements, the thermal and mechanical components of energy (and hence the physical parameters associated herewith, such as the temperature, entropy, stress, strain, density, etc.) may vary.

The method started explicitly taking into account the porosity and the nanocrystalline structure. In doing so, the algorithms for calculating the local moments of forces and the angular velocities of microrotations to arise in structurally heterogeneous medium have been implemented. The suggested model of heat transfer in a discrete medium takes into account the influence of heterogeneity of the internal structure of a solid on the propagation pattern of heat flows.

\section{DESCRIPTION OF THE TEMPERATURE FIELD CALCULATION METHOD IN TERMS OF THE EXCITABLE CELLULAR AUTOMATA METHOD}

Within the concept of the excitable cellular automata, the space occupied by the simulated model is divided into elementary cells (active elements) to form a network of a cellular automaton. Besides the state, each element is characterized by numerical parameters corresponding to the simulated volume of a medium. At the zero step of the numerical experiment, the initial values of temperature, strain, thermal conductivity, specific heat, and the coefficient of linear thermal expansion are specified for each element. Then, for each time step $(n)$, a new value of element temperature is calculated taking into account the thermal flows from each neighboring element:

$$
T_{i}^{n}=T_{i}^{n-1}+\frac{1}{c_{i} \rho_{i} V} \sum_{k=1}^{N} Q_{i k}^{n},
$$

where $T_{i}^{n-1}, T_{i}^{n}$-temperatures of the $i$-th element for the $(n-1)$-th and $n$-th time steps, $c_{i}$ - the heat capacity of $i$-th element, $\rho_{i}$ - the density of the $i$-th element, $V$ - the volume of an element, $Q_{i k}^{n}$ - the flow of thermal energy from a neighboring element (with $k$ index) into the researched one with index $i$ for the $n$-th time step, $N$-the number of neighbors.

A change in thermal energy $Q_{i k}^{n}$ is calculated via Fourier law:

$$
Q_{i k}^{n}=\frac{\lambda_{i k} \Omega}{l}\left(T_{k}^{n-1}-T_{i}^{n-1}\right) \Delta \tau \text {. }
$$

Here $\lambda_{i k}$-the factor of thermal cross conductivity, $l$ - the distance between the centres of the elements, $\Omega$ - the area of an adjacent element edge, $\Delta \tau$ - the time step value. In a simple case, the coefficient of thermal cross conductivity could be calculated as follows:

$$
\lambda_{i k}=\left(\lambda_{i}+\lambda_{k}\right) / 2 .
$$

Here $\lambda_{i}, \lambda_{k}$ - the factors of heat conductivity of $i$-th and $k$-th elements, and

$$
\lambda_{j}=\tilde{\lambda}_{j} e^{-\varphi_{j} \theta_{i k}}(j=i, k) \text {, }
$$

where $\tilde{\lambda}_{j}$-the factor of thermal conductivity of $j$-th automaton, $\varphi_{j}$ - the factor that determines the degree of phonon influence on the material's thermal conductivity, $\theta_{i k}$ - the angle (in degrees) that is determined by the difference in the crystalline lattices' orientations of $i$-th and $k$-th automaton (in the current version of the program, $\left.\theta_{i k}=\psi_{i}-\psi_{k}\right)$.

As soon as new temperature values $T_{i}^{n}$ are obtained from formula (1), the following novel values for each $i$-th element are calculated:

a) Change in strain due to thermal expansion:

$$
\Delta_{Q} \varepsilon_{i}^{n}=\alpha_{i}\left(T_{i}^{n}-T_{i}^{n-1}\right) ;
$$

b) Corresponding changes in the stresses and the mechanical energy $A_{i}^{n}$ :

$$
\Delta_{Q} \sigma_{i}^{n}=\alpha_{i} \cdot\left(T_{i}^{n}-T_{i}^{n-1}\right) \cdot Y_{i}^{n},
$$




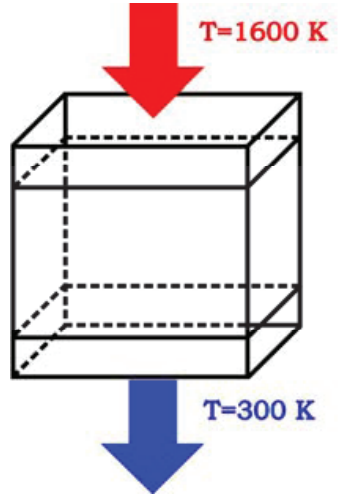

(a)

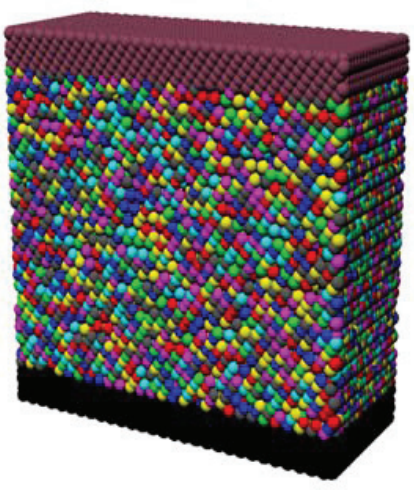

(b)

FIGURE 1. Specimen loading scheme (a) and general appearance of the specimen (b)

$$
\Delta_{Q} A_{i}^{n}=\frac{Y_{i}^{n} V_{C A}}{2}\left[\operatorname{sign}\left(\varepsilon_{i}^{n-1}+\Delta_{Q} \varepsilon_{i}^{n}\right)\left(\varepsilon_{i}^{n-1}+\Delta_{Q} \varepsilon_{i}^{n}\right)^{2}-\operatorname{sign}\left(\varepsilon_{i}^{n-1}\right)\left(\varepsilon_{i}^{n-1}\right)^{2}\right]
$$

c) New value of thermal energy $Q_{i}^{n}$ :

$$
Q_{i}^{n}=Q_{i}^{n-1}+c_{i} \cdot\left(T_{i}^{n}-T_{i}^{n-1}\right)-\Delta_{Q} A_{i}^{n}
$$

d) According to (8), the temperature value is amended:

$$
T_{i}^{n}=T_{i}^{n}-\frac{\Delta_{Q} A_{i}^{n}}{c_{i}} .
$$

Thus, a fraction of the change in the element's heat energy is spent on changing its mechanical energy due to the process of thermal expansion. As a result, internal thermal stresses are accumulated which are transformed into mechanical energy flows redistributed over the elements network. The algorithms for simulating mechanical energy flows within the excitable cellular automata method is discussed in detail in [3].

The heat transfer method was tested by comparing the simulation results with the analytical solutions for a onedimensional rod and a three-dimensional parallelepiped. Thus, the average value of the relative error in the temperature calculation does not exceed $0.1 \%$.

Based on the excitable cellular automata method and the computer program, some numerical experiments on the thermal loading of the two three-layered compositions with the intermediate layers of different structures have been carried out. The specimen sizes were $5 \times 5 \times 2 \mathrm{~mm}$, while the diameter was $0.1 \mu \mathrm{m}$. The upper ceramic layer had a thickness of $0.5 \mu \mathrm{m}$; the intermediate layer with thickness of $4 \mu \mathrm{m}$ was of modified nanostructured alumina; the layer bottom one with the thickness of $0.5 \mu \mathrm{m}$ was composed of coarse-grain copper. The grain size in the intermediate layer was set at 0.5 and $0.1 \mu \mathrm{m}$. The effect of the porosity presence for each type of specimens was investigated. The upper face was heated up to $1600 \mathrm{~K}$. The temperature at the bottom one was set constant $(300 \mathrm{~K})$ that imitated substrate cooling. The loading scheme and the general appearance of the specimens are shown in Fig. 1.

Based on the results of the numerical experiments, the graphs of the volumetric distribution of the force moment values averaged over the mass (Fig. 2(a)) and effective stress (Fig. 2(b)) for both compositions were plotted. Under the effective stress, the elastic energy specific over the volume value is defined here.

As seen from the graph in Fig. 2(a), the maximum values of the force moments are achieved for the specimen with the intermediate nanoporous sublayer with the grain size of $0.1 \mu \mathrm{m}$. This speaks in favor of the assumption that a significant part of the energy of internal thermal stresses is spent on the microvortices formation that gives rise to the dissipation of elastic energy and its transfer into the entropic component of the total stored energy. This assumption is confirmed by the analysis of the graph shown in Fig. 2(b). It is evident that the pore-free structures possess low moments of force (Fig. 2(a)), whereas in the porous structure with the grain size of $0.1 \mu \mathrm{m}$, the lowest level of stress at the "intermediate layer-substrate" interface takes place (Fig. 2(b)).

This suggests that the nanoporous structure has the best relaxation ability among all specimens under study. 


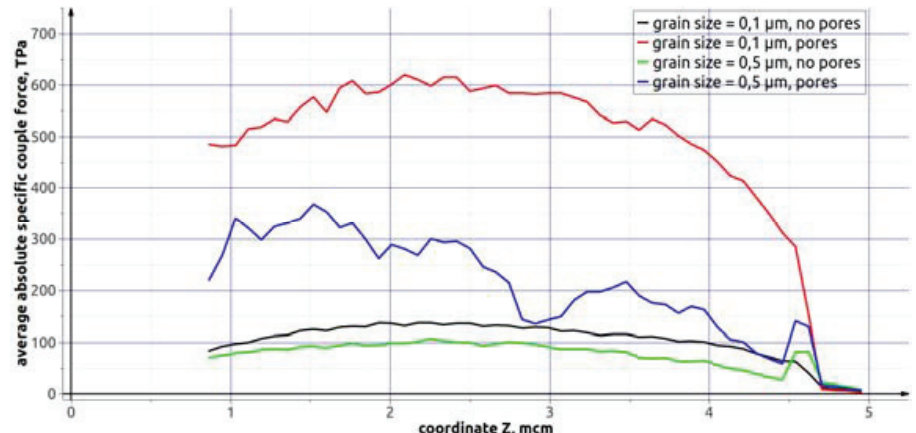

(a)

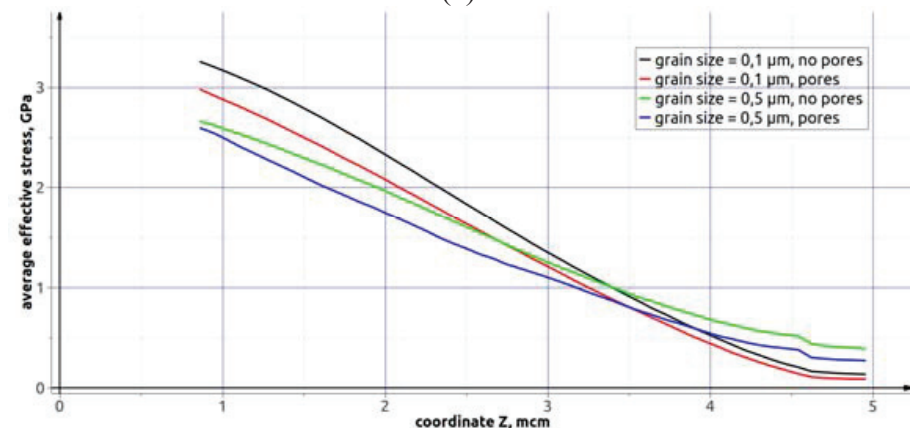

(b)

FIGURE 2. Distribution of the absolute values of force moments averaged over the mass (a) and effective stresses (b) in the depth of the examined compositions at the end of the numerical experiment.

The $Z$-axis corresponds to the depth of the intermediate layer

\section{CONCLUSIONS}

A new modification of the multilevel hybrid discrete-continuous approach of excitable cellular automata has been developed. The new version of the method takes into account the nanocrystalline structure and porosity of the material in an explicit form. Thanks to the explicit taking into account the local rotations and angular velocities of microrotations that arise in a structurally heterogeneous medium, it became possible to quantify the level of the energy dissipation of various structures under thermal impact. It is shown that the stress level at the "coatingsubstrate" interface in the nanoporous structure is significantly reduced due to the rise of microrotations.

Thus, the theoretical studies have shown that nanostructuring of intermediate sublayer and introducing nanoporosity there ensures a positive effect on the ability of stress relaxation in the thermal barrier coating. All simulation results are in good agreement with the experiment.

\section{ACKNOWLEDGEMENTS}

The Study was supported by projects of the RAS Presidium Program No. 25, the Russian Foundation for Basic Research (Nos. 14-01-00789, 13-01-00403, 13-08-00616 and 13-08-90402) and the Grant of the President of the Russian Federation for the Support of Leading Scientific Schools (NSh 2817.2014.1).

\section{REFERENCES}

1. V. E. Panin, V. P. Sergeev, and A. V. Panin, Nanostructuring of Surface Layers of Structural Materials and Deposition of Nanostructured Coatings (Izd. TPU, Tomsk, 2008).

2. V. E. Panin and V. E. Egorushkin, Phys. Mesomech. 14(5-6), 207 (2011).

3. D. D. Moiseenko, Yu. I. Pochivalov, P. V. Maksimov, and V. E. Panin, Phys. Mesomech. 16(3), 248 (2013). 